Cao, X., Liu, J., Jiang, N., and Chen, Q. 2014. "Particle image velocimetry measurement of indoor airflow field: A review of the technologies and applications," Energy and Buildings, $69,367-380$

\title{
Particle Image Velocimetry measurement of indoor airflow field: A review of the technologies and applications
}

Xiaodong Cao ${ }^{\mathrm{a}}$, Junjie Liu ${ }^{\mathrm{a},{ }^{*}}$, Nan Jiang ${ }^{\mathrm{b}, \mathrm{c}}$, Qingyan Chen ${ }^{\mathrm{a}, \mathrm{d}}$

${ }^{a}$ School of Environmental Science and Engineering, Tianjin University, Tianjin 300072, China

${ }^{\mathrm{b}}$ School of Mechanical Engineering, Tianjin University, Tianjin 300072, China

${ }^{\mathrm{c}}$ Tianjin Key Laboratory of Modern Engineering Mechanics, Tianjin 300072, China

${ }^{\mathrm{d} S c h o o l}$ of Mechanical Engineering, Purdue University, West Lafayette, IN 47907, USA

*Email address: jjliu@tju.edu.cn

\begin{abstract}
Quantifying the airflow field in building room or vehicle cabin indoor space is crucial for creating a thermal comfortable and healthy indoor environment. Airflow field measurement can provide quantitative information of indoor air distribution and local air velocity around occupants or passengers, which has strong relationship with the ventilation effectiveness, the pollutant transportation and the energy conservation in a building or a vehicle. However, the airflow field in indoor environment is normally characterized by high turbulent level and unsteady due to relatively low air velocity from the diffuser, thermal plume of heat source and unsteady perturbation of occupants' behavior. Generally, it is very difficult to conduct accurate and detailed measurement of such a complex turbulent flow field with point-wise anemometry. Therefore, using Particle Image Velocimetry (PIV) is a promising technique to solve this problem. This paper firstly gave an introduction of the typical PIV technologies used in indoor environment measurement, and then summarized the state-of-the-art applications of PIV in measuring the indoor airflow fields. The overview shows that PIV has gradually become the most popular and versatile tools for measuring various indoor airflow fields. The quantitative and detailed turbulent flow information obtained by PIV is critical for analyzing turbulent properties and validating numerical simulations. Specifically, the authors focused on the pros and cons of PIV measurement and gave the typical parameters of PIV used in indoor airflow field measurements. Generally, the researchers should pay more attention to the selection of appropriate PIV system parameters according to their specific research needs. The accuracy of PIV measurement was also discussed. Nevertheless, PIV is not always the optimum tool for indoor airflow measurements. Measurement systems using commercial PIV are often restricted to the block of optical paths and the size limit of image. It is necessary to develop large spatial scale optical system and camera shooting technology to overcome these limitations.
\end{abstract}


Keywords: Indoor environment; Airflow; Particle Image Velocimetry (PIV); Measurement Technology

\section{Introduction}

Ventilation concepts, including natural ventilation and mechanical ventilation, have been widely used and discussed in indoor environment quality studies [1-4]. The performance of ventilation system can be assessed in terms of airflow pattern, the distribution of contaminant concentration, air velocity, air temperature, global ventilation effectiveness and air distribution effectiveness, etc [4]. Among the various studies dealing with the performances and applications of ventilation systems, the indoor airflow patterns and airflow field are some of the most widely concerned issues. It is because that the air motion responsible for transporting both heat and pollutants is one of the main factors controlling indoor environment quality, which helps to create a thermally comfortable and safe built environment as well as to provide adequate ventilation for reducing gaseous and particulate concentrations of contaminant. Moreover, indoor air distribution designs in HVAC also have a strong influence on building energy consumptions. For a space heating and cooling system, the type of air distribution system plays a decisive role in the energy performance. A reasonable approach to indoor air distribution, such as the under floor air distribution (UFAD), can save a great amount of energy due to the higher supply air temperature and lower ventilation rate. A previous study [5] showed that the potential energy benefit of UFAD is significant compared to the conventional ceiling air distribution system. Therefore, quantifying the airflow field in a room is crucial for creating both a thermal comfortable, healthy and energy-saving indoor environment.

Experimental measurements and numerical simulations are the two main methods available for the indoor airflow field studies. Airflow field measurement can provide reliable quantitative information of indoor air distribution or local air velocity around occupant [6-8]. However, experimental measurement is usually difficult and time-consuming. Numerical simulation can provide both global and detailed airflow quantities under various cases at relatively low cost, which is very helpful for the design and assessment of indoor ventilation system. However, conducting numerical simulation always relies on accurate measurements as the boundary conditions [9-11]. The simulated results also cannot be completely trusted unless being validated by corresponding high quality experimental data [12-15]. Therefore, experimental measurement is still the necessary and fundamental step of indoor airflow study.

Nevertheless, the airflow field in indoor environment is normally characterized by high turbulent level and unsteady flow due to the relatively low air velocities from diffusers, thermal plumes of heat sources and unsteady perturbation of occupants' behavior. Therefore, it is not easy to conduct accurate airflow field measurement in indoor environment. Sun and Zhang [16] summarized various kinds of modern indoor air motion measurement techniques. In general, the velocimetry can be divided into point-wise and global-wise. The point-wise techniques include Pitot tube and 
rotating-vane anemometry, which are based on pressure differential principles; hotwire anemometry, hot-film anemometry and hot-sphere anemometry, on heat transfer principles; ultrasonic anemometry (UA), on acoustics principles; and Laser Doppler Velocimetry (LDV), on Doppler shift principles. The global-wise techniques mainly include Particle Tracking Velocimetry (PTV), Particle Streak Velocimetry (PSV) and Particle Image Velocimetry (PIV), which are based on optical principles. The traditional point-wise anemometry can only obtain the velocity information at the point of the probe sensor. Generally, it is very difficult to conduct accurate and detailed measurement of the global turbulent airflow field in indoor environment with point-wise velocimetry. Meanwhile, most of the point-wise velocimetry, such as thermal anemometry and ultrasonic anemometry, are thought to have perturbations on the local airflow. Even using the non-intrusive point-wise techniques (e.g. LDV), it is still excessively difficult and time-consuming to conduct the global airflow field measurement point by point.

For this reason, optical velocimetry have gradually become the alternative tools for measuring indoor airflow distributions. Optical velocimetry can obtain the velocities and related statistical information in a global domain without disturbing the airflow, which makes the whole instantaneous and mean velocity field measurement possible. PIV is the most robust and widely used optical velocimetry for flow field studies [17, 18]. Specifically, PIV has begun to be used in indoor airflow field measurements during the last decade. The main reasons for the popularity of PIV measurements are well-developed technologies, rich experimental experiences and commercially available systems. Though the setups of PTV and PSV are similar to PIV and have been applied in some indoor air motion measurements [19-23], they are still developing and not commercially available in the market at present, due to the complex algorithms and special experimental settings involved. It is not convenient for common researchers in HVAC\&R field to use PTV or PSV to conduct the indoor airflow field measurements. Therefore, PIV is supposed to be the most appropriate and promising technique for airflow field measurement in indoor environment.

This study focuses on providing an overview of the typical PIV technologies used in indoor environment and the state-of-the-art applications of PIV in measuring the indoor airflow fields. The key technologies used in typical PIV systems and the pros and cons of PIV measurement in indoor environment will be reviewed during the last decade. The accuracy of PIV measurement and the limits of measurement systems using PIV will be discussed.

\section{PIV technologies for indoor application}

The term 'Particle Image Velocimetry' (PIV) first appeared in the literature in 1980s [18]. The significant scientific and technical progress achieved in the last 30 years in lasers, image recording and evaluation techniques, and computer techniques led to the further development of PIV technique. Presently, PIV has become a powerful tool for studying flow field in indoor environment. The measurement principle and major developments of PIV have been reviewed in many excellent 
papers [24-28] and in a comprehensive book by Raffel et al. [17]. Therefore, in this section, we just introduce the PIV technologies focusing on the applications in indoor environment.

PIV is a technique to achieve quantitative measurement of instantaneous turbulent velocity fields as an extension of qualitative flow visualization. PIV tracks the tracer particles seeded in the air to obtain the whole velocity field of the given measurement area. A typical PIV system consists of a multi-pulsed laser system, one or more digital cameras synchronized with the lasers, and a computer to control the system and analysis the data. A 2D-2C PIV system only uses one camera while a 2D-3C PIV system uses two cameras. As for volumetric PIV system, such as tomographic PIV system, more than three cameras will be used. Due to the complicated experimental setup and much more expensive price of a volumetric PIV system, the most common used PIV in indoor airflow measurements is still 2D-2C PIV. The basic setup of a 2D-2C PIV system is shown in Fig. 1 [29]. The key technologies applied in a typical 2D-PIV system will be briefly discussed hereinafter.

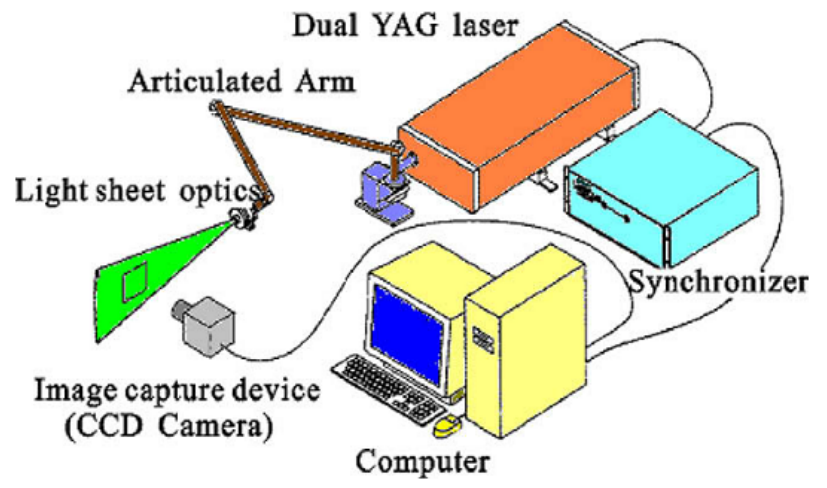

Fig. 1. Schematic of a 2D-2C PIV measurement system [29].

\subsection{Illumination system}

For the indoor airflow field measurements, the most widely used illumination system is a double-pulsed Nd:Yag lasers system with an articulated delivery arm to generate a green light sheet of $532 \mathrm{~nm}$ wavelength. The light sheet optics locate at the end of the arm can be oriented at any angle to generate a light sheet and illuminate the tracer particles in the measured region of interest. The main component of light sheet optics is one or more cylindrical lens to focus the light sheet to request thickness and field angle. To guarantee the measured flow field can keep in a plane, the thickness of the light sheet in the measurement area is typically about 1-3mm [16] or less. However, using such a thin light sheet as the illumination method also brings about a challenge for measuring a strong three-dimensional flow field in a sizeable indoor environment. In this case, some particles recorded by the cameras in the first frame may move out of the measured plane and cannot be captured in the next frame. That will limit the accuracy of PIV measurement to the regions of the thin plane flow [30].

For this reason, an important parameter to set when using lasers as the illumination 
source is the time delay between the pulses, $\Delta \mathrm{t}$. The time delay should not only be long enough to determine the displacement of the tracer particles between the two pulses, but also need to be short enough to avoid particles leaving out of the light sheet between subsequent illuminations. Cao et al. [31,32] gave a recommendation of $\Delta t$ in the study of attached plane jet based on the optimization of particle shift in image under normal room conditions. According to their research, the separation of the particle images (in pixels) should be larger than the accuracy of the peak detection and smaller than a quarter of the selected interrogation area size. Therefore, the optimum pulse delay depends on the desired interrogation area size $d_{\text {Int }}$ (in pixels) and on the maximum velocity $U_{\max }$ in airflow fields recorded, consequently will be in the range of:

$$
\frac{\text { pixel }}{U_{\max }}<\Delta t=\frac{\Delta x}{U_{\max }}<\frac{1}{4} \frac{d_{I n t}}{U_{\max }}
$$

Li et al. [29] presented an easier method to determine $\Delta t$. They found a practical relation (Eq. (2)) between $\Delta \mathrm{t}$ and the maximum velocity of the shooting section $\left(U_{\max }\right)$. In particular, $\Delta \mathrm{t}$ increases with the decrease of the maximum velocity of the shooting section. The Eq. (2) is applicable to the similar PIV experiments.

$$
U_{\max } \times \Delta t(\mu s)=250
$$

Another critical factor about the light source selection is the desired measuring area. For instance, the full-scale experiments require large illuminated areas. However, typical Nd:Yag lasers used in indoor airflow measurements are only available with the power up to $500 \mathrm{~mJ}$ per pulse. In this case, by using oil droplets with a diameter of 1 $\mu \mathrm{m}$ as the tracer particles, the measuring area will be restricted to $1 \mathrm{~m}^{2}$, which is apparently insufficient for a full-size room. Therefore, to achieve large scale measurement, a practical approach is to use conventional light sources combined with larger tracer particles. Different types of conventional light sources, such as halogen lamps [33], flash lamps or large arrays of LEDs have been proved to be able to form a light sheet with the thickness of several centimeters using cylindrical lenses. The advantage of the conventional light sources is the large illuminated area while the disadvantage is the relatively low light intensity. However, using neutrally buoyant large size tracer particles which have higher light reflection ability can compensate for this disadvantage. Besides, one should pay attention to the heat gain effect on the airflow when using conventional light sources.

\subsection{Image recording devices}

The most common image recording devices used in indoor airflow measurements are Coupled Charged Devices (CCD) cameras and Complementary Metal Oxide Semiconductor (CMOS) cameras. CCD cameras have been widespread used in PIV experiments for their increased spatial resolution, convenience data transmission and 
image processing, minimum exposure time, high light sensitivity at $532 \mathrm{~nm}$ and low background noise. In general, a CCD element is an electronic sensor which can convert photons into electrons. A CCD sensor in the camera normally consists of an array of many individual CCD elements, which also called pixels. Today, commercially available CCD cameras typically has the sensor resolution range from $2 \mathrm{M}$ (1600 pixels $\times 1200$ pixels $)$ to $29 \mathrm{M}$ (6576 pixels $\times 4384$ pixels), and the corresponding frame frequency from $35 \mathrm{~Hz}$ to $2 \mathrm{~Hz}$ [34]. Thus, one should make a trade-off between the spatial resolution and the temporal resolution, and select the CCD cameras depend on certain applications. For example, a high resolution CCD camera is necessary for the large-scale measurement in a full-size room, which aims to obtain the complete airflow structures. Contrarily, a high frequency CCD camera is more suitable for studying the small-scale turbulent characteristics of airflows. The dynamic range of CCD sensors should also be considered to evaluate the signal quality per pixel. Normally, a dynamic arrange of 8 or 10 bits data output per pixel is sufficient for most PIV purposes. However, with the advanced cooling technique, a 14 or 16 bit camera is also available for the applications such as Planar Laser-Induced Fluorescence (PLIF) where very low noises and high dynamic range are required.

When it comes to the time-resolved measurement for acquiring accurate turbulent information, using a high-speed CMOS camera is a better choice than a CCD camera. High-speed recording based on recently developed CMOS sensors even allows for acquisition in the $\mathrm{kHz}$ range, which is very promising for turbulence study. Using a CMOS sensor allows for the recording and handling of up to a few thousand frames per second at acceptable noise levels, but in trade of the sensor resolution. Though as a more advanced image recording technique, the low spatial resolution has become the main obstacle for CMOS cameras to completely replace the CCD cameras. This critical drawback limits the applications of CMOS cameras only to small-scale measurements. Thus, CCD cameras are still the main image recording devices for indoor PIV measurement currently due to the better image quality and wider applied range. A detailed comparison between CCD cameras and CMOS cameras is reported by Hain et.al [35].

\subsection{Tracer particles}

Tracer particles play a key role in indoor PIV measurements, because PIV actually measures the velocities of tracer particles instead of real airflow velocities. Generally, the size of tracer particles should be not only small enough to achieve good tracking behavior of the turbulence but also large enough to scatter sufficient light signal for image recording devices.

\subsubsection{Properties of the tracer particles}

The tracking behaviors of tracer particles are particularly crucial for PIV measurement accuracy. However, completely neutrally buoyant particles for airflow are still not found due to the low air density. In fact, the densities of most widely used 
particles for indoor PIV measurements, such as the oil droplet, are much larger than the air. Therefore, the influence of gravitational forces cannot be neglected considering the significant density difference. The gravitationally induced velocity $u_{g}$ can be derived from the Stokes' drag law (Eq. (3)), which is considered to apply when using the assumption of spherical particles in a viscous fluid at a low Reynolds number. This condition holds true for most indoor PIV applications.

$u_{g}=d_{p}^{2} \frac{\left(\rho_{p}-\rho_{f}\right)}{18 \mu} g$

Where $\mathrm{g}$ is the gravitational acceleration, $\rho_{f}$ and $\mu$ are the density and dynamic viscosity of the air, $\rho_{p}$ and $d_{p}$ are the density and diameter of the particles. Eq. (3) indicates that the diameter of the not neutrally buoyant particles should be small enough to reduce the velocity lag between particles and airflow induced by gravitational effect. Stokes' drag law gives a preliminary estimation of the tracking behavior of the particles. Nevertheless, the Stokes' drag law may underestimate the actual velocity lag in the airflow with high turbulence level. The maximum allowable particle size decreases with increasing airflow velocity, turbulent level and velocity gradients.

Seeding the tracer particles into the airflow with an initial velocity $u_{p 0}$ may also lead to some perturbations on the original airflow field. For PIV applications, the particle's response to the turbulent airflow can be quantified by the relaxation time of the particles:

$\tau_{p}=d_{p}^{2} \frac{\rho_{p}}{18 \mu}$

Thus, the difference between particles velocity $u_{p}$ and air velocity $u_{f}$ is determined by the time $t$ and the relaxation time of the particles:

$u_{f}-u_{p}=u_{g}+\left(u_{f}-u_{p 0}-u_{g}\right) \exp \left(-\frac{t}{\tau_{p}}\right)$

We can conclude from Eq. (5) that the effect of initial particle seeding velocity can be neglected only when $t$ is much longer than $\tau_{p}$. In addition, the tracking behavior of tracer particles can be easily evaluated by using Stokes number (St). St is regarded as the ratio of the particle response time to the time scale $\tau_{f}$ of the airflow:

$S t=\frac{\tau_{p}}{\tau_{f}}$

For room airflows, the St of tracer particles is recommended to be less than 0.1 .

Besides, the scattering characteristics of tracer particles also need to be considered, especially for the large-scale PIV measurements. The scattering characteristics of the 
particles can be quantified by the scattering cross section $C_{s}$, which is a function of the ratio of the particle diameter to the laser wavelength for spherical particles. Table 1 compares the approximate $C_{s}$ for a diatomic molecule and two larger particles [36]. It is clear that larger particles can give exponential stronger light signals, which relate to the larger measuring area and higher signal-to-noise ratio.

Table 1 The scattering cross section as a function of the particle size [36].

\begin{tabular}{ll}
\hline Particle diameter & Scattering cross section $C_{s}$. \\
\hline Molecule & $\approx 10^{-33} \mathrm{~m}^{2}$ \\
$1 \mu \mathrm{m}$ & $\approx 10^{-12} \mathrm{~m}^{2}$ \\
$10 \mu \mathrm{m}$ & $\approx 10^{-9} \mathrm{~m}^{2}$ \\
\hline
\end{tabular}

In sum, the size of the tracer particles should be optimized to make a balance between the tracking behavior and the scattering characteristics. In general, the acceptable tracking ability is more crucial for the study of turbulent or high speed airflow in a small region, such as the inlet air jet; while the scattering demand should be more concerned when studying the air distributions in a full-size room. Particularly, a larger particle size is feasible by using helium-filled-soap bubbles, which have a relatively low density ratio to air. More detailed discussions on the properties of the tracer particles can be found in the literatures [17, 36, and 37].

\subsubsection{The generation and seeding of typical particles}

The tracer particles used in indoor environment should be harmless to human and environmental protection. Oil or DEHS droplets, theatrical fog and helium-filled-soap bubbles are the most widespread used for indoor airflow measurements. Oil or DEHS droplets are very promising particles for small-scale turbulence measurements, because of their good tracking ability and high stability. Theatrical fog fluid generally consists of a proprietary blend of glycol/glycerin and water. The generated fog is more suitable for continuous local seeding due to its high concentration, uniformity and plenty generation amount. Helium-filled-soap bubbles (HFSBs) are typically used in large-scale PIV measurements [37, 38], for their neutrally buoyant properties and much higher scattering efficiency than other particles.

Various methods are available for the generation of the particles to seed indoor airflow $[39,40]$. Ideally, the generators should produce particles with a monodisperse size distribution and at a constant high enough production rate to meet the spatial resolution requirement of PIV experiment. For indoor applications, condensation and atomization are the two most widely used generation methods. The most common condensation aerosol is a fog machine, which is feasible to generate large amount of droplets or theatrical fog. Fog machine uses either an inert gas or an electric pump to propel mineral oil, glycol, or glycol and water mixture into a heat exchanger, where the solution is vaporized [41]. The vaporized fluid is then discharged through the nozzle into the airflow where, upon mixing with the cooler air, it is condensed into an aerosol consisting of millions of fine particles. Commercial theatrical fog machines 
are typically inexpensive and they generate plenty of particles. However, the generation rate of this kind of generators tends to be unsteady and discontinuous due to the limit of heating capability. It is generally not easy to conduct long time continuous measurement using fog machines. Another popular generation method for oil or DEHS droplets is atomized by Laskin nozzle generators [42]. Laskin atomizers use pressurized air jets to draw up the liquid in the reservoir to produce air bubbles containing atomized particles. The particles are then released into the airflow when the bubbles reach the surface. The size of the atomized particles is normally small due to an impactor plate for retaining big particles. The generation rate of Laskin atomizers is controllable and continuous, but smaller than the fog machines. In fact, for all types of generators, the trade-off between quality and concentration of the particles should be made. Besides, the HFSBs are atomized by orifice type nozzle using bubble soap solutions combined with pressurized helium and air. Both commercial generators and special-designed generators [37] are available for producing HFSBs.

Particles should be seeded into airflow with sufficient, steady and spatial uniform concentration. Generally, a higher seeding concentration is preferred for better measurement spatial resolution. However, excessively high density seeding may lead to poor spatial resolution due to bad scattering performance. The ideal concentration of the tracer particles is 10 to 20 particles in an interrogation area [43]. A uniform seeding size is also desirable in order to avoid excessive intensity from larger particles and background noise and incoherent signals from small particles. It is recommended to use a particle counter to analyze the particle size distribution during the measuring process. The seeding method can be divided into global seeding and local seeding. Global seeding is feasible in small-scale model or re-circulate wind tunnel to achieve whole velocity field measurement. However, global uniform seeding in large size space may be restricted to the generation capability. If only a part of the airflow field is of interest in a large space, local seeding is more feasible. If local seeding is adopted, the position of seeding probes should be carefully positioned to achieve suitable particle concentration without disturbing local airflow.

\subsection{Image evaluation methods}

It is clear from the principle of PIV that this technique is based on the direct determination of the two fundamental dimensions of the particle velocity: displacement and time. However, it is impossible to directly calculate the velocity for every particle due to the high concentration and the overlaps between particles. Therefore, image evaluation methods are necessary to derive the displacement information from raw particle images. The preferred evaluation method in PIV is to capture two images on two separate frames, and perform multistep cross-correlation analysis. This cross-correlation function has a significant peak, providing the magnitude and direction of the velocity vector without ambiguity. The correlation methods are commonly based on digital Fast Fourier Transform (FFT) algorithms for calculating the correlation functions. 
For indoor applications, the recently most widespread used evaluation method is adaptive correlation. The adaptive correlation method [44] iteratively calculates velocity vectors with an initial interrogation area (IA) of the size $\mathrm{N}$ time the size of the final IA and uses the intermediary results as information for the next IA of smaller size, until the final IA size is reached. IA is a sub-area in the recorded images and its dimensional setting directly determines the spatial resolution and accuracy of the measurement. The smaller IA size and higher overlap ratio can achieve higher spatial resolution, but require higher quality image recordings and consume longer computing time. According to the reviewed papers, the size of IA is typically set to be 32 pixels $\times 32$ pixels or 64 pixels $\times 64$ pixels with overlaps of $50 \%$ or $25 \%$ for indoor PIV applications. In addition, the adaptive correlation method can achieve higher accuracy supplemented with high sub-pixel accuracy and adaptive deforming window algorithm. Currently, adaptive correlation is available in most of the commercial PIV software packages.

Adaptive PIV interrogation [44, 45] is a more advanced and automatic correlation algorithm for calculating velocity vectors based on particle images. The method will iteratively adjust the size and shape of the IA in order to adapt to local seeding densities and flow gradients. The method also includes options to apply window functions, frequency filtering as well as validation in the form of universal outlier detection [46]. In general, adaptive PIV can achieve higher accuracy and spatial resolution results than adaptive correlation but consuming much more computing sources. Another advanced evaluation method potential for indoor applications is 2D or 3D Least Squares Matching (LSM) [47-48]. In contrast to correlation based methods, LSM is a gray-level tracking technique, which performs translation, deformation and rotation of the IA [47]. The LSM algorithm iteratively compares gray value information of an IA in the first time step with the gray value information in the second time step. This is an iterative least squares procedure applying affine transformations on the IAs. Thus, LSM can not only calculate the zero order translational velocities just like the correlation methods, but also simultaneously take the first order terms of fluid motion into account. For this reason, LSM can obtain a more accurate calculation of the velocity gradient tensor, the rotation tensor and the deformation tensor without any assumptions and manipulations. However, LSM received much less attention than correlation methods because much longer computation times and requirement of extremely high quality particle images.

Though having distinct advantages, the two advanced methods were less used in indoor PIV experiments. Nevertheless, these methods have great potentials for the fine measurement of complex indoor airflow, such as the inlet air jet and wall turbulence, where larger velocity gradient tensor exists and greater accuracy is needed. Unfortunately, though variety kinds of algorithms available, there is not a single algorithm that has the best performance everywhere [26]. Detailed analyses of the performances of the state-of-the-art evaluation methods are available in the main results of PIV challenges [25-27].

In summary, various techniques are involved in a typical PIV system for indoor airflow field measurements. However, no universally applicable PIV system is 
available for different applications. In practice, many compromises and decisions have to be made on a case by case basis. Table 2 gives the pros and cons of three typical 2D-2C PIV systems that the authors have used in indoor airflow measurements. The researchers should pay more attention to the selection of appropriate PIV system parameters for their specific needs, such as the measuring area, the spatial and temporal resolution and the required accuracy. The above overview does not include the principles for volumetric PIV techniques, such as stereoscopic PIV, tomographic PIV and defocusing PIV [49], because these techniques are still not well developed for indoor measurement applications. However, volumetric PIV systems have indeed been used in a small portion of the indoor airflow studies, including the measurements of air jets and airflows in the breathing zone. It is sure that the volumetric PIV will become the mainstream indoor velocimetry in the future.

Table 2 Typical 2D-PIV systems used in indoor airflow field measurements

\begin{tabular}{|c|c|c|c|c|}
\hline PIV system & Main parameters & Advantage & Disadvantage & Applicability \\
\hline TR-PIV & $\begin{array}{l}10 \mathrm{MJ} \text { Lasers } \\
\text { with } 1 \mathrm{M} \text { CMOS } \\
\text { camera }\end{array}$ & $\begin{array}{l}\text { Very High time } \\
\text { resolution }(2 \mathrm{kHz}) ; \\
\text { High spatial } \\
\text { resolution }\end{array}$ & $\begin{array}{c}\text { Restricted FOV } \\
(0.15 \mathrm{~m} \times 0.13 \mathrm{~m}) ; \\
\text { Bulky }\end{array}$ & $\begin{array}{c}\text { Small-scale } \\
\text { model }\end{array}$ \\
\hline Mini-PIV & $\begin{array}{c}\text { 5MJ Lasers } \\
\text { with } 0.3 \mathrm{M} \text { CMOS } \\
\text { camera }\end{array}$ & $\begin{array}{c}\text { High time } \\
\text { resolution }(200 \mathrm{~Hz}) ; \\
\text { Portable }\end{array}$ & $\begin{array}{c}\text { Restricted FOV } \\
(0.08 \mathrm{~m} \times 0.06 \mathrm{~m}) \\
\text { Low spatial } \\
\text { resolution }\end{array}$ & $\begin{array}{l}\text { Critical area in } \\
\text { large space } \\
\text { (e.g. air inlet) }\end{array}$ \\
\hline $\begin{array}{c}\text { High } \\
\text { Power PIV }\end{array}$ & $\begin{array}{c}\text { 350MJ Lasers } \\
\text { with } 11 \mathrm{M} \mathrm{CCD} \\
\text { camera }\end{array}$ & $\begin{array}{c}\text { High spatial } \\
\text { resolution; } \\
\text { Large } \\
\operatorname{FOV}(0.9 \mathrm{~m} \times 0.6 \mathrm{~m})\end{array}$ & $\begin{array}{l}\text { Low time } \\
\text { resolution }(3.5 \mathrm{~Hz}) ; \\
\text { Bulky }\end{array}$ & $\begin{array}{l}\text { Full-scale } \\
\text { model }\end{array}$ \\
\hline
\end{tabular}

\section{Applications in indoor airflow field measurements}

In this section, the applications of PIV techniques in indoor airflow measurements were summarized. We mainly focused on the publications in English language journals in the past decade within the applied range including ventilation devices, human airflows and indoor airflow distributions. The review may be not inclusive but represent the status and trend of the indoor PIV applications.

\subsection{Ventilation devices}

Ventilation devices play an important role as the air supply boundary conditions for the ventilated rooms, which are very crucial for creating thermally comfortable indoor environment with acceptable indoor air quality. Besides, energy efficiency has 
become one of the most concerned design criteria for building appliance. Thus, it is critical to design effective and energy-saving ventilation devices for indoor environment. However, the airflows generated by ventilation devices are usually complex and difficult to be measured. Conventional velocimetry cannot fulfill the measurement requirement of the complex airflows generated by ventilation devices, such as the jet flows. In contrast, PIV is an ideal measurement tool to investigate the jet dynamic behavior, such as the spatial vortex structure and the turbulent transitional process.

Cao et al. [31, 32] used PIV to reveal the structure of a turbulent attached plane jet in the entrainment process of the ambient air downstream from an active chilled beam in a climate chamber. The PIV system was capable of measuring the attached plane jet at a low Reynolds number with high turbulence intensity quantitatively and instantaneously. Fig. 2 shows their PIV measurements of the jet velocity fields at different slot average velocities. The results provided a precise visualization of the structure of the attached jet during the turbulent transition and entrainment process after leaving the supply slot. During the measurement, it was observed that the optimization of the measurement parameters, such as the time delay between pulses and the seeding density, has to be performed with extreme caution. Meslem et al. [50] employed PIV system to acquire instantaneous spatial velocities distributions of two different kinds of jet. The results showed that cross-shaped jets performed much better than the circular reference jets as a method of passive control of air diffusion systems for buildings. They also used PIV to measure the flow field of a turbulent twin cross-shaped jet on a perforated panel diffuser [51]. The experimental results were used to validate various numerical models. The results showed that the SST k- $\omega$ model would appear to be the most appropriate tool for the prediction of parallel lobed jets in perforated panel optimization. Elvsén and Sandberg [52] used PIV to study the entrainment into the buoyant jet close to a diffuser for displacement ventilation. The results showed a small, approximately constant entrainment at the upper side of the jet. Montoya et al. [53] demonstrated the application of synthetic jet actuators for controlling indoor air quality in a dedicated closed chamber that included a simulated ventilation system. The flow field in the room was determined using PIV. The effectiveness of the synthetic jet-based flow control was demonstrated for controlling airflow and aerosol dispersion and removal in the closed chamber. Nastase et al. [54] employed PIV system to measure the flow fields from two different kinds of grills. The result showed that jet flows from innovative grilles with lobed ailerons ensure higher mixing in a room than baseline jets from commercial grilles with straight ailerons. Nino et al. [55] used PIV to measure the velocity flow field generated by a submerged rectangular air, simulating the air curtain jet. The measurements could capture the homogeneous velocity distribution in the exit section of the nozzle and the formation of vortex structure instability.

The above studies suggest that PIV can provide detailed information about the complex air jets generated by various kinds of ventilation devices, which would have been impossible with conventional point-wise measurements. The data acquired by PIV are suitable for the validation of numerical simulations and for the development 
of the empirical models and basic theories of air jets. Nevertheless, the measurement of air jets using PIV is not easy. During the measurement, the seeding of particles has to be performed with extreme caution. Generally, it is suggested to inject oil droplets or theatrical fog into the upstream of the air inlet with appropriate seeding density. Both insufficient and excess particle concentrations would lead to poor results.

(a)

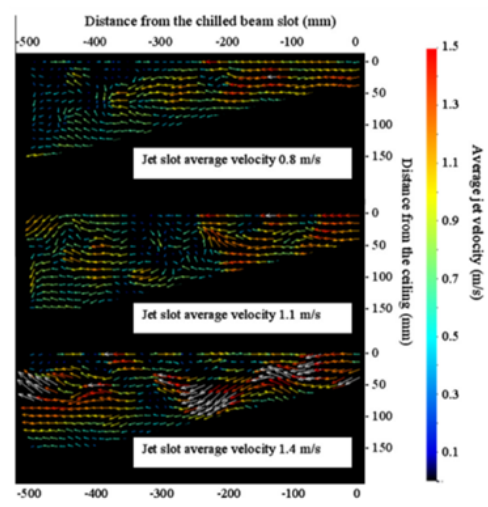

(b)

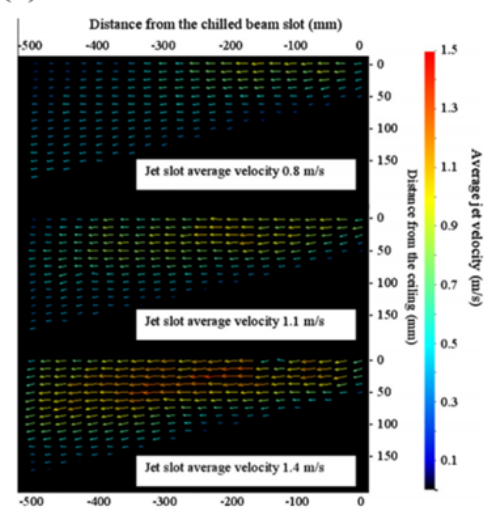

Fig. 2. PIV measurements of a turbulent attached plane jet discharged from an active chilled beam in a room by Cao et al. [32]: (a) instantaneous jet velocity vector fields, (b) time averaged jet velocity vector fields

Besides, PIV also can be used to measure the flow fields inside the ventilation devices. It is generally very difficult to study the dynamic and thermal behavior of the flows inside the ventilated cavities with conventional methods. In this case, PIV is a more suitable technique for its non-intrusive property. Sanjuan et al. [56, 57] used PIV to measure the velocity field inside the air cavity of an Open Joint Ventilated Facades (OJVF) model, as shown in Fig. 3(a). Fig. 3(b) shows the seeding density inside the ventilated cavity during the experimental procedure and Fig. 3(c) presents the measured velocity vectors inside the cavity. The comparison between experimental and numerical data indicated that the k- $\varepsilon$ two equation turbulence models and the DO radiation models showed best fittings in velocity profiles. Chami and Zoughaib [58] used PIV system to characterize the buoyancy-driven air flow inside the thermosyphon. A numerical model representing the natural convection within the thermosyphon was also developed. The predicted velocity distributions and the induced mass flow rate were in good agreement with the PIV experimental results.

These investigations demonstrate that the PIV is a very effective tool to investigate the performance of ventilation devices. It is also found that most of these studies were based on 2D-PIV systems. In fact, the airflow fields generated by many devices, such as air conditioners, usually have significant three dimensional flow structures. Thus, it is more desired to use volumetric PIV to conduct detailed measurements of these flows [50-51, 59]. 
(a)

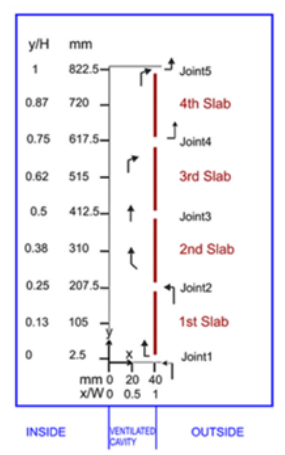

(b)

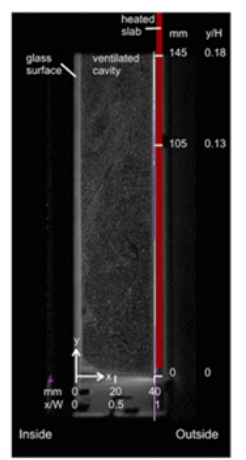

(c)

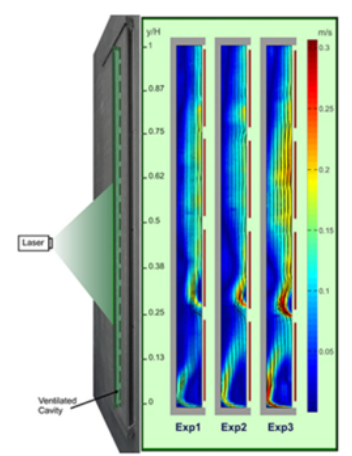

Fig. 3. PIV measurement of the velocity field inside the air cavity of an OJVF model by Sanjuan et al. [56]. (a) The ventilated cavity dimensions of the OJVF model. (b) Seeding density inside the ventilated cavity. (c) Velocity vectors measured in the experiments.

\subsection{Human airflows}

Knowledge of the behaviors and physics of human airflows, such as thermal plumes, coughing flows and breathing flows, are very critical to create high indoor environment quality. For example, the design of an effective ventilation system typical hinges on the flow rate of human thermal plume; while the airflow information of the respiratory activities, such as coughing and sneezing, are important for understanding the infection mechanism of airborne transmission diseases and quantifying the exposure to indoor pollutants. However, it is very difficult to capture the instantaneous (e.g. coughing and sneezing) and unsteady (e.g. thermal plume) human airflows by point-wise velocimetry. Thus, PIV is certainly more suitable for measuring the human airflows.

Özcan et al. [60] obtained mean velocity data around the head of a real-life size breathing thermal manikin for two cases of 'no breathing' and 'continuous exhalation' through nose by using PIV. Marr et al. [61] presented stereoscopic PIV measurements to analysis downstream of the inlet along a thermal manikin in order to simulate the interaction of an inlet duct with the heated body of an average indoor occupant. They also employed TR PIV and stereoscopic PIV to obtain the quantitative velocity information in the breathing zone of a thermal manikin placed within a chamber with simulated breathing [62-63]. The results suggested that the airflow due to breathing is both unsteady and highly anisotropic. Common steady and isotropic assumptions made in the simulations were not admissible. Eisner et al. [64] used PIV to study the complex particle flows induced by the foot motion. The visualization of the complex flows using a sequence of PIV images was useful to understand how foot motion affected air transport and thus how walking affected contaminant dispersion.

Specifically, above measurements were based on thermal manikins. However, the airflow around a real human body may much differ from a manikin. It would be more meaningful to conduct measurements based on human volunteers to study the physics of human airflows or obtain accurate boundary conditions for further simulations. Craven et al. [65] used PIV to characterize the velocity field around a human 
volunteer in the temperature-stratified room. The results were then compared to computational fluid dynamics (CFD) simulation results. Though employed a highly simplified model of the human form, the simulation results compared quite well with the PIV data. Zhu et al. [66] used PIV to measure the velocity distribution around the mouth of the coughing subject with flour as the tracer particle. Chao et al. [67] presented the PIV data of expiration air jet velocities from volunteers during coughing and speaking. The average expiration air velocity was $11.7 \mathrm{~m} / \mathrm{s}$ for coughing and $3.9 \mathrm{~m} / \mathrm{s}$ for speaking. Similarly, the initial velocity and angle of exhaled airflow from coughing and speaking was measured with 26 tested subjects using PIV by Kwon et al. [68]. VanSciver et al. [69] also applied PIV system to measure the velocity field of human cough from 29 subjects. Cough flow velocity profiles, average widths of the cough jet, and maximum cough velocities were obtained. Nevertheless, it is worth mentioning that the laser beam in PIV system is a safety hazard and may harm eyes and skin. Thus, the PIV experiments involving human volunteers should be paid great attention to the safety problems.

The recent applications indicate that the PIV measurements of human airflows are becoming popular and sophisticated. Also the trend in this area is to use human volunteers instead of manikins. If using volunteers as the subjects, the tracer particles should be non-toxic and similarly represent the particles expelled from a human's mouth during breathing and coughing. In this case, theatrical fog seems a good choice for the measurements of thermal plumes [65] or coughing flows [69]. Fig. 4(a) and Fig. 4(b) show the examples of these two investigations respectively.

(a)

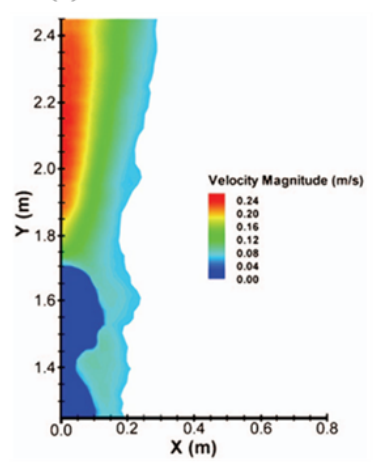

(b)

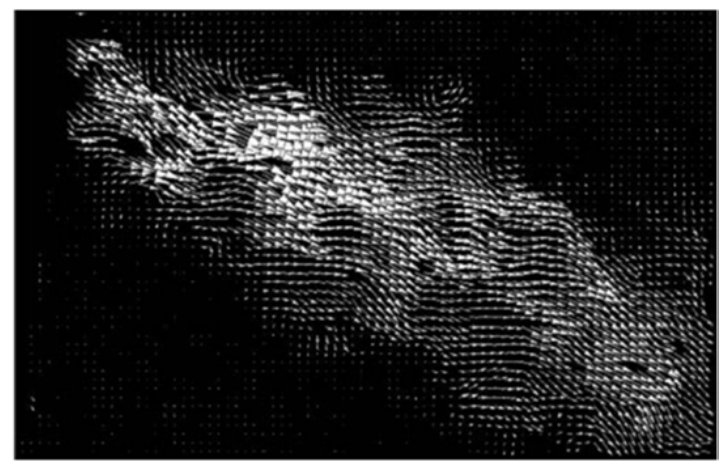

Fig. 4. PIV investigations of actual human airflows. (a) Velocity magnitude contours of human thermal plume in the sagittal plane obtained by Craven et al. [65]. (b) Velocity field of a typical cough flow measured by VanSciver et al. [69].

\subsection{Indoor airflow fields}

\subsubsection{Small-scale models}

The small-scale experimental models use measuring techniques to predict or evaluate ventilation performance with a reduced scale of the buildings or rooms [3]. Generally, small-scale models are very suitable for PIV measurements. The 
dimensions of small-scale models are similar to the field of view of typical PIV systems and the enclosures can be easily made transparent. Therefore, PIV could straightforwardly measure the airflow field in the whole cross section in a small-scale model. Besides, the controllable boundary conditions of small-scale models could be changed for studying different cases.

Li et al. [29] used PIV to study the air distribution in the model of a powerhouse. It would be impossible to conduct such a measurement in a full-scale model. Therefore, a 1/50 scale-down model was built up to mimic the real building. Their research found that the supply air velocity and the air inlet location had a remarkable effect on air distribution characteristics of the large space and occupant zone velocity. Hooff et al. [70] presented detailed PIV measurements and an analysis of transitional flow in a ventilated enclosure model shown in Fig. 5(a). Fig. 5(b) depicts the time-averaged velocity in the entire cross section of the model for $\mathrm{Re}=1000$. Specific attention was given to the Coanda effect by which the free plane jet develops into a wall jet. Kang and Lee [71] investigated the natural ventilation of entrained air inside a scale-down factory building using a louver ventilator. The mean and fluctuating velocity fields were measured using PIV technique. Karava et al. [72] employed the PIV technique for the evaluation of the air velocity field in a generic single-zone building model with cross-ventilation under isothermal flow conditions. It was found that airflow patterns in buildings with cross-ventilation are complex and cannot be predicted by simplified macroscopic models such as the orifice equation. Rouaud et al. [73] made an assessment on the influence of external perturbations on an original minienvironment. The PIV measurements were initially carried out in order to describe the steady flow in the minienvironment. Posner et al. [74] compared the results from numerical simulations with PIV experimental measurements of indoor air flows in a one-tenth down-scale model room. The results showed that the simulations predicted the measured trends in a model room very well, with relative error not much larger than 20\%. Kobayashi et al. [75] conducted experimental investigation and CFD analysis of cross-ventilated flow through single room detached house model. They used PIV to measure the velocity distributions on the windward and leeward side to investigate differences in these flow patterns due to the size of the opening. The CFD simulated results showed relatively well agreement with PIV data. Poussou et al. [76] conducted PIV and PLIF measurements on a one-tenth scale, water-based model to investigate the effects of a moving body on airflow and contaminant transport inside an airliner cabin. Liu et al. [77] used PIV and CFD to investigate multiple flow structures in a three-dimensional scale chamber with opposed jets. Pedersen and Meyer [78] used PIV to obtain velocity data in a domain covering a square region near the inlet of a scale model of a ventilated room. Proper Orthogonal Decomposition (POD) was used to analyze dominant flow structures. 
(a)

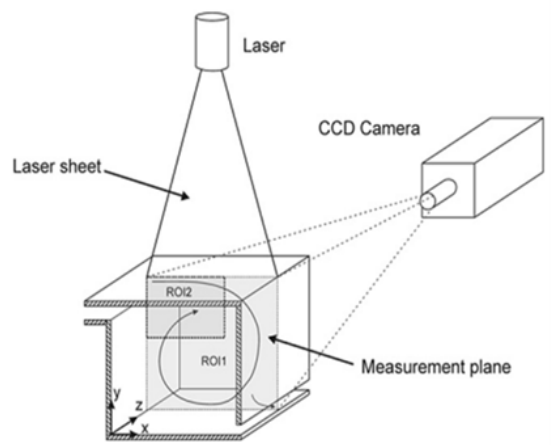

(b)

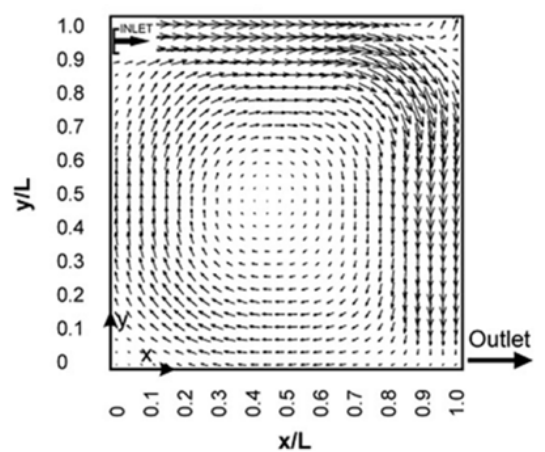

Fig. 5. PIV measurements of the transitional airflow in a small-scale model conducted by Hooff et al. [70]. (a) PIV set-up. (b) Time-averaged velocity vector fields for $\mathrm{Re}=1000$ in the entire cross-section.

Various applications indicate that PIV is popular and appropriate for small-scale model experiments. However, small-scale models may suffer from scaling problems associated with combined modeling of inertial and buoyancy forces, and simplified geometries. Therefore, the small-scale results may not accurately represent the real conditions and difficult to be directly used to investigate ventilation performance. Nevertheless, PIV measurements in small-scale models are still important. In fact, our literature review found that the high quality data obtained by PIV in small-scale models were very useful to validate numerical simulations.

\subsubsection{Full-scale models}

Measurements of the airflows in full scale models are also critical for analyzing the ventilation system performances and validating numerical simulations. Compared with small-scale models, full-scale models can better represent the real conditions but more expensive and complex. Generally, the applications of PIV in full-scale models are much more difficult than small-scale models due to the large space, non-transparent enclosures and inside obstructions. However, great efforts have been made to achieve PIV measurements in full-scale models and the results are reviewed as follow.

\subsubsection{Room environment}

The research group led by Yuanhui Zhang has performed many large-scale PIV measurements in a full-scale test room $(5.5 \times 3.7 \times 2.4 \mathrm{~m})$. They developed both large-scale 2D-PIV system [33] and large-scale stereoscopic PIV system [79] to obtain full-scale room airflow fields for validating RNG $\mathrm{k}-\varepsilon$ model and LES model $[80,81]$. Helium-filled bubbles of neutral buoyancy were used to seed room airflow and a bank of projector lamps were used to offer illumination. Fig. 6(a) shows an example of their measurement result by 2D-PIV system. As shown in Fig. 6(a), the full-scale PIV results can directly give the realistic prediction of the indoor air 
distribution without scaling and simplification problems. Their studies suggested that the modified PIV technique can be a practical method to quantitatively measure the indoor air velocity fields in entire cross sections. Besides the large-scale applications, PIV were also used to obtain local airflow fields in real size rooms. Hosni et al. [82] used PIV to characterize the airflow field around a standing person in a simple room with realistic and well-documented environmental conditions. Caciolo et al. [83] studied the flow field and the air exchange rate generated by single-sided ventilation in a full-scale room. PIV was used to measure the flow field on a vertical plane orthogonal to the opening in front of it, as depicted in Fig. 6(b). Mortensen et al. [84] performed a PIV investigation of the airflow pattern in the microclimate behind furniture placed next to exterior walls. The results indicated that the flow rates behind the furniture would increase with increased distance between the closet and the wall, and even higher airflow rates were seen when the furniture was elevated by legs. The above applications indicate that using PIV in full-scale rooms seems to be less popular in recent years, because it is much more convenient to obtain the airflows in entire cross sections using small-scale models. In full-scale model investigations, the research emphasis was also switched to local airflows rather than entire flow patterns. Nevertheless, it is still meaningful to conduct large-scale PIV measurements in full-scale rooms, for they can give the most real predictions of ventilation performances in buildings.

(a)

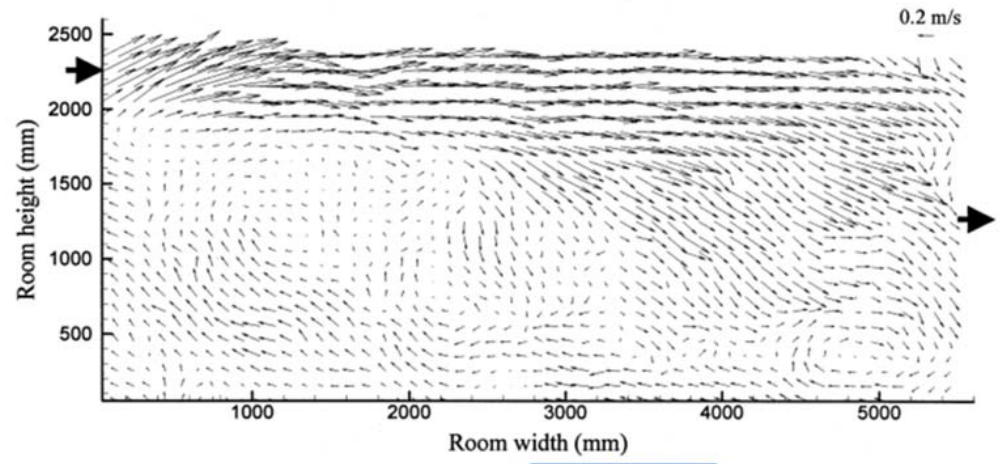

(b)

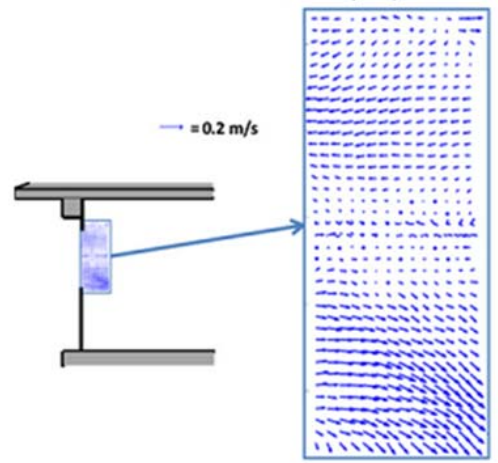

Fig. 6. PIV measurements in full scale models. (a) Airflow patterns in a full-scale room for cross-flow ventilation at $19.5 \mathrm{ACH}$ measured by Zhao et al. [33]. (b) Measure of the flow field in front of the opening by Caciolo et al. [84]. 


\subsubsection{Cabin environment}

Unlike room environment, the applications of PIV in cabin environment still focused on the full-scale models. The reasons for this trend are the complex diffusers, geometries and inside obstructions of the cabins, which are very difficult to scale down. The small-scale cabin models may suffer from scale-similarity problems and their results could not be directly used. Yang et al. [85] used PIV to obtain the flow field in an empty car cabin mockup installed in a thermostatic chamber. The PIV measurement was performed at a total of 18 regions within the section. Lee et al. [86] conducted PIV investigation on the ventilation flow inside the passenger compartment of an empty real commercial automobile under operating conditions. Günther et al. [87] and Bosbach et al. [88] used PIV to measure the airflow in an empty A380 aircraft cabin mockup (Fig. 7(a)). Lin et al. [89] also applied stereoscopic PIV to measure air velocity field in a generic empty aircraft cabin and used the data to validate the LES (Large Eddy Simulation) results. Their numerical predictions reasonably agreed with the experimental data. However, the airflow pattern in an empty cabin can be significantly different from an occupied cabin. Therefore, it is much more promising to obtain experimental data in an occupied cabin mockup. Kühn et al. [90] performed large scale PIV measurement of the airflow patterns in an occupied cabin section of the A380 upper deck. Wan et al. [91] and Sze et al. [92] used PIV to characterize the airflow pattern at 4 selected locations in an occupied double-aisle aircraft cabin to validate numerical simulations. The above PIV measurements in occupied aircraft cabins could only reach the upper part of the cabins as the seats and manikins severely blocked the optical paths, as shown in Fig. 7(b). Mo et al. [93] employed PIV to obtain the relatively complete velocity and turbulence intensity profiles of the airflow inside a section of an occupied aircraft cabin. But they lowered many seat-backs to clear the optical paths for the PIV measurement, which still made the geometrics they studied not represented the actual conditions well. Therefore, the PIV measurement regions in occupied cabin environment are often restricted by inside obstructions due to the technology limitation.

Our review of the literatures found that PIV has indeed become the mainstream measuring technique for the studies of air distributions in various cabin mockups. However, great attention should be paid to the restricted measurement area in occupied situation. How to overcome this limitation is the key issue in further studies.

(a)

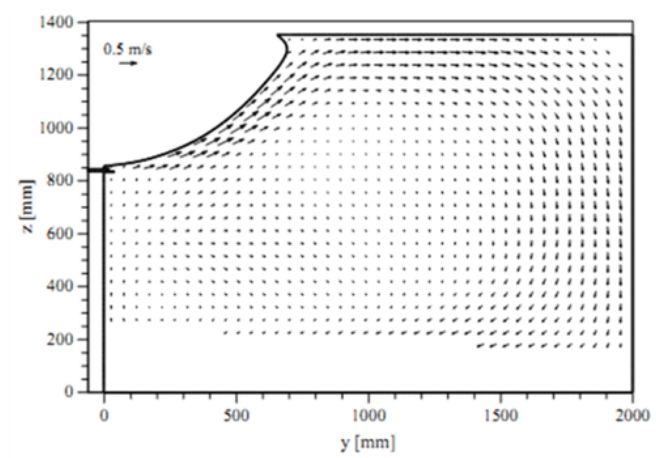

(b)

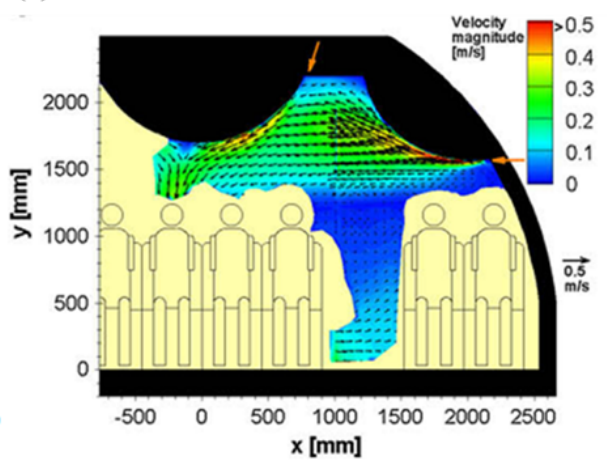


Fig. 7. Measured flow fields in aircraft cabins with PIV. (a) Measure of the isothermal flow field in an empty A380 mockup by Bosbach et al. [88]. (b) Velocity distribution under cooling conditions in a full-occupied A380 mockup obtained by Kühn et al. [90].

\section{Discussion}

It is noticeable that velocity measurements are not free from errors, especially for the low-velocity measurements in room environment [94]. Unlike other anemometers, PIV is a measuring technique whose accuracy is directly determined by both the displacement error and the uncertainty in the time delay between pulses. The uncertainty in the time delay between pulses could be minimized by using the methods mentioned in section 2.2 or trials in the experimental procedure.

In contrast, the displacement error is a prior uncertainty source for indoor PIV applications and complex to estimate, because PIV measurement itself can be seen as a chain of error producing steps. Firstly, the tracer particles follow the turbulent flow according to their density, size, mass and shape, which directly determine the deviation between the measured velocities and real air velocities. Then the image acquisition and evaluation procedure produces its own errors. The accuracy of calculated velocity data mainly depend on the quality of particle image, size of interrogation area and the statistical correlation method [30, 94-96]. Moreover, the expertises of the researcher also influence the measurement accuracy. The experimental setup and procedure of a PIV system is much more complex than other techniques. A sophisticated technician can apply the PIV system into the measured airflow more properly than a green hand. An accepted conclusion has yet been made on the overall accuracy of PIV applications in indoor environment.

Sandberg [97] indicated that the relative error can be estimated to be on the order of $1-2 \%$ in a two dimensional PIV system for indoor applications. In another study, it was reported that an accuracy of 5\% to $10 \%$ could be achieved for the room airflow measurements using the two-component PIV technique [16]. In an investigation of a turbulent twin cross-shaped jet on a perforated panel diffuser [51], the maximal displacement error for the 2D PIV measurements was estimated to be $1.4 \%$. For the stereoscopic PIV results, the maximal displacement error was equal to $0.8,1.7$ and $2.2 \%$ for the longitudinal, vertical and transversal directions respectively. A more practical way to validate the PIV measurement accuracy is to compare the PIV measurements with other anemometers at certain points in the experimental procedure. Zhao et al. [33] concluded that their PIV measurements in a full-scale room generally agree with the cross hotwire measurements in terms of air velocity profiles at specific locations. However, some differences existed due to turbulent properties of indoor airflow. Cao et al. [31, 32] compared the measurements of an attached plane jet obtained with PIV and hot-sphere anemometers. Fig. 8 shows that the difference between the measured velocities by PIV and by anemometers was larger than $20 \%$ in the inner layer and out layer. The measured maximum jet velocities are closer, less than $20 \%$ difference, by two measurement methods. It is worth noting that the accuracy of the hot-sphere anemometer at low velocity is also on the order of $10 \%-20 \%$ [16]. These results 
indicate a fact that the estimations of PIV measurement accuracy are on a case by case basis. However, it is reasonable to estimate the overall accuracy as approximately $10 \%$ at most by above investigations, which is sufficient for normal indoor applications. The accuracy could also achieve $2 \%$ or less for precision measurement by carefully designed experiment.
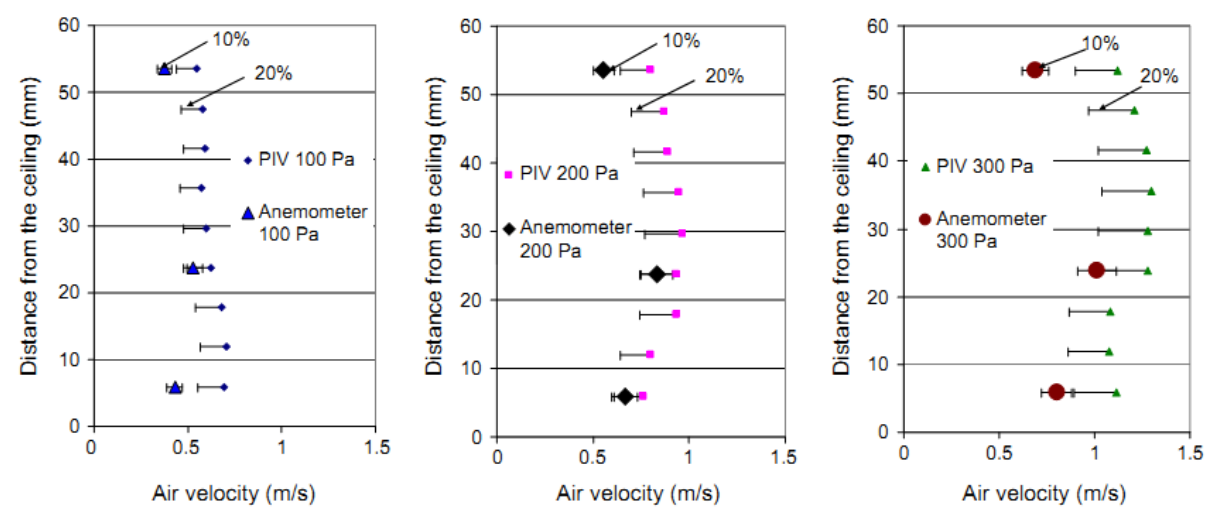

Fig. 8. A comparison of the measurements by PIV and hot-sphere anemometers [32].

Besides the errors produced by the PIV system itself, the uncertainty in the time-averaged behavior also merit attention. The uncertainty in the ensemble average PIV data describing turbulent flows is mainly dependent on the sample size and the order of statistics. Ullum et al. [98] investigated the effect of sample size on the accuracy of time-averaged PIV data for a single point in a grid-generated turbulence in its early decay. It was concluded that 100 vector maps for a component of mean velocity were required for an uncertainties of averages at a given point to be within $1 \%$ for a flow of $10 \%$ turbulence intensity. However, 20000 vector maps were needed for a component of normal stress with this level of accuracy. The study demonstrated the need for larger sample sizes for higher order statistics. Uzol and Camci [99] studied the effect of sample size on the accuracy of ensemble averaged PIV data inside the wake region of a 2-row staggered array of circular cylinders. They concluded that the PIV measurement errors in the ensemble averaged data increased not only with the increasing turbulence intensity levels but with the decreasing velocity magnitude as well. Stafford et al. [100] also analyzed the significance of the number of vector maps recorded on time-averaged statistics using PIV for a practical application of a radial fan flow.

Theoretical standard error estimates and an empirical bootstrapping technique [101] had been implemented for determining high confidence levels in the accuracy of the time-averaged quantities for the entire region of interest. The results indicated that a trade-off between sample size and accuracy may be necessary using this measurement technique. It can be concluded from these studies that sufficient uncorrelated PIV sample size had to be acquired in order to obtain reliable time-averaged turbulent quantities, particularly for higher order statistics, such as turbulence intensity and Reynolds stress. However, so far little literature is available for the time-averaged behavior of PIV for indoor airflow measurement, which should be further studied. In 
addition, the turbulent scale and the time period of indoor airflow may also have influence on the measuring frequency and sampling size, which is another interesting area of further study.

Though PIV is a powerful tool for indoor airflow investigation, it also has several serious limitations. Firstly, measurement systems using PIV are often restricted to the block of optical paths, particularly for full-scale models. Though transparent materials could be used to solve the light obstruction problem, it will lead to the difficulty in maintaining the thermal boundaries. Moreover, the obstructions using transparent materials inside the models may change the optical paths and distort the particle images. Therefore, PIV is not the optimum tool to measure the airflow field in the whole cross section in a highly obstructed indoor space.

Secondly, the measurement area of commercial PIV is often limited due to the insufficient laser intensity, the low-spatial resolution and the restricted space to expand the optical light sheet. Since this critical drawback limits the applications of PIV in full-scale models, it is necessary to develop large scale optical system and camera shooting technology to overcome this limitation. So far, a few non-commercial large-scale PIV systems have already been applied to indoor environment, which typically use HFBS as the tracer particles and conventional light $[33,79]$ or large expanded laser light sheet [38] as the illumination sources.

Furthermore, a PIV system is expensive, bulky, and difficult to use. Accurate PIV measurement of indoor airflow requires a relatively significant investment in equipment, time and experimental expertise. In many engineering applications, especially for in-situ measurements, conventional anemometers are more practical than PIV systems. Only one in-situ PIV measurement [84] can be found in all reviewed articles. More portable and easy-to-use PIV systems are crucial for extending the indoor PIV applications. The Mini-PIV listed in Table 2 is a good example for the development trend of PIV. In addition, PIV is not always the optimum choice when conducting detailed turbulence investigations. For instance, if one want to study the turbulent energy spectrum of the inlet airflow, a high-frequency hot-wire anemometer is obvious a better option than a PIV system.

\section{Conclusions}

The key technologies involved in a typical PIV system for indoor airflow field measurements were discussed in sequence. However, no universally applicable PIV system was found for different applications. In practical use, many compromises and decisions should be made. The researchers have to pay more attention to the selection of appropriate PIV system parameters according to their specific needs.

The reviewed publications revealed that PIV has gradually become the most popular and versatile tools for measuring various indoor airflow fields. The quantitative and detailed flow visualization information obtained by PIV is critical for analyzing turbulent properties and validating numerical simulations. PIV is a very effective tool to investigate the performance of ventilation devices. Volumetric PIV is suggested for the further investigations of the three dimensional airflow structures 
induced by the devices with complex geometries. PIV is also suitable to measure the human airflows. The safety hazard should be paid great attention for the PIV experiments involving human volunteers. For indoor PIV measurements, small-scale models are the most appropriate and convenient. However, small-scale models may suffer from scaling problems and not represent the real conditions. In contrast, it is much more difficult to perform PIV measurements in full-scale models. Even so, full-scale PIV measurements are still very important, for the data obtained from full-scale models are the most realistic and reliable. In addition, the accuracy of PIV measurement is complex to determined, but sufficient for indoor applications.

Nevertheless, PIV is not always the optimum tool for indoor airflow measurements. Measurement systems using commercial PIV are often restricted to the block of optical paths and the size limit of image. It is necessary to develop large scale optical system and camera shooting technology to overcome these limitations. More portable and practical PIV system with lower cost is also desired in the future.

\section{Acknowledgments}

The research presented in this paper was financially supported by the National Basic Research Program of China (The 973 Program) through grant No. 2012CB720100.

\section{References}

[1] Kato S, Ito K, Murakami S. Analysis of visitation frequency through particle tracking method based on LES and model experiment. Indoor Air. 2003;13(2):182-93.

[2] Niachou K, Hassid S, Santamouris M, Livada I. Experimental performance investigation of natural, mechanical and hybrid ventilation in urban environment. Building and Environment. 2008;43(8):1373-82.

[3] Chen Q. Ventilation performance prediction for buildings: A method overview and recent applications. Building and Environment. 2009;44(4):848-58.

[4] Chen Q, Lee K, Mazumdar S, Poussou S, Wang L, Wang M, et al. Ventilation performance prediction for buildings: model assessment. Building and Environment. 2010;45(2):295-303.

[5] Lim TS, Schaefer L, Kim JT, Kim G. Energy Benefit of the Underfloor Air Distribution System for Reducing Air-Conditioning and Heating Loads in Buildings. Indoor and Built Environment. 2012;21(1):62-70.

[6] Chao C, Wan M. Airflow and air temperature distribution in the occupied region of an underfloor ventilation system. Building and Environment. 2004;39(7):749-62.

[7] Krajč́́k M, Simone A, Olesen BW. Air distribution and ventilation effectiveness in an occupied room heated by warm air. Energy and Buildings. 2012;55:94-101.

[8] Morsing S, Strøm J, Zhang G, Kai P. Scale model experiments to determine the effects of internal airflow and floor design on gaseous emissions from animal houses. Biosystems Engineering. 2008;99(1):99-104.

[9] Chen Q, Srebric J. A procedure for verification, validation, and reporting of indoor environment CFD analyses. HVAC\&R Research. 2002;8(2):201-16. 
[10] Srebric J, Vukovic V, He G, Yang X. CFD boundary conditions for contaminant dispersion, heat transfer and airflow simulations around human occupants in indoor environments. Building and Environment. 2008;43(3):294-303.

[11] Liu W, Wen J, Chao J, Yin W, Shen C, Lai D, et al. Accurate and high-resolution boundary conditions and flow fields in the first-class cabin of an MD-82 commercial airliner. Atmospheric Environment. 2012;56:33-44.

[12] Zhang Z, Zhang W, Zhai ZJ, Chen QY. Evaluation of various turbulence models in predicting airflow and turbulence in enclosed environments by CFD: Part 2-Comparison with experimental data from literature. HVAC\&R Research. 2007;13(6):871-86.

[13] Kuznik F, Rusaouen G, Brau J. Experimental and numerical study of a full scale ventilated enclosure: Comparison of four two equations closure turbulence models. Building and Environment. 2007;42(3):1043-53.

[14] Rohdin P, Moshfegh B. Numerical predictions of indoor climate in large industrial premises. A comparison between different $\mathrm{k}-\varepsilon$ models supported by field measurements. Building and Environment. 2007;42(11):3872-82.

[15] Wang M, Chen Q. Assessment of Various Turbulence Models for Transitional Flows in an Enclosed Environment (RP-1271). HVAC\&R Research. 2009;15(6):1099-119.

[16] Sun Y, Zhang Y. An overview of room air motion measurement: technology and application. HVAC\&R Research. 2007;13(6):929-50.

[17] Raffel M, Willert CE, Wereley ST, Kompenhans J. Particle image velocimetry: a practical guide: Springer; 2007.

[18] Adrian RJ. Twenty years of particle image velocimetry. Experiments in Fluids. 2005;39(2):159-69.

[19] Wang A, Zhang Y, Sun Y, Wang X. Experimental study of ventilation effectiveness and air velocity distribution in an aircraft cabin mockup. Building and Environment. 2008;43(3):337-43.

[20] Rosenstiel M, Grigat R-R. Segmentation and classification of streaks in a large-scale particle streak tracking system. Flow Measurement and Instrumentation. 2010;21(1):1-7.

[21] Biwole PH, Yan W, Zhang Y, Roux J-J. A complete 3D particle tracking algorithm and its applications to the indoor airflow study. Measurement Science and Technology. 2009;20(11):115403.

[22] Zhao L, Wang X, Zhang Y, Riskowski G. Analysis of airflow in a full-scale room with non-isothermal jet ventilation using PTV techniques. ASHRAE Transactions. 2007;113(Part 1):414-25.

[23] Lobutova E, Resagk C, Putze T. Investigation of large-scale circulations in room air flows using three-dimensional particle tracking velocimetry. Building and Environment. 2010;45(7):1653-62.

[24] Grant I. Particle image velocimetry: a review. Proceedings of the Institution of Mechanical Engineers, Part C: Journal of Mechanical Engineering Science. 1997;211(1):55-76.

[25] Stanislas M, Okamoto K, Kähler C. Main results of the first international PIV challenge. Measurement Science and Technology. 2003;14(10):R63.

[26] Stanislas M, Okamoto K, Kähler C, Westerweel J. Main results of the second international PIV challenge. Experiments in Fluids. 2005;39(2):170-91.

[27] Stanislas M, Okamoto K, Kähler C, Westerweel J, Scarano F. Main results of the third international PIV challenge. Experiments in Fluids. 2008;45(1):27-71. 
[28] Dabiri D. Digital particle image thermometry/velocimetry: a review. Experiments in Fluids. 2009;46(2):191-241.

[29] Li A, Qin E, Xin B, Wang G, Wang J. Experimental analysis on the air distribution of powerhouse of Hohhot hydropower station with 2D-PIV. Energy Conversion and Management. 2010;51(1):33-41.

[30] Hart DP. PIV error correction. Experiments in Fluids. 2000;29(1):13-22.

[31] Cao G, Sivukari M, Kurnitski J, Ruponen M, Seppänen O. Particle Image Velocimetry (PIV) application in the measurement of indoor air distribution by an active chilled beam. Building and Environment. 2010;45(9):1932-40.

[32] Cao G, Sivukari M, Kurnitski J, Ruponen M. PIV measurement of the attached plane jet velocity field at a high turbulence intensity level in a room. International Journal of Heat and Fluid Flow. 2010;31(5):897-908.

[33] Zhao L, Zhang Y, Wang X, Riskowski G, Christianson L. Measurement of two-dimensional air velocities in a full-scale room using particle image velocimetry. ASHRAE Transactions. 2001;107(2):434-44.

[34] DYNAMICS D. FlowSense EO camera series. wwwdantecdynamicscom 2012.

[35] Hain R, Kähler CJ, Tropea C. Comparison of CCD, CMOS and intensified cameras. Experiments in Fluids. 2007;42(3):403-11.

[36] Melling A. Tracer particles and seeding for particle image velocimetry. Measurement Science and Technology. 1997;8(12):1406.

[37] Meyers JF. Generation of particles and seeding. VKI for Fluid Dynamics, Lecture Series 1991-08, Laser Velocimetry. 1991.

[38] Bosbach J, Kühn M, Wagner C. Large scale particle image velocimetry with helium filled soap bubbles. Experiments in Fluids. 2009;46(3):539-47.

[39] Kühn M, Ehrenfried K, Bosbach J, Wagner C. Large-scale tomographic particle image velocimetry using helium-filled soap bubbles. Experiments in Fluids. 2011;50(4):929-48.

[40] Kähler C, Sammler B, Kompenhans J. Generation and control of particle size distributions for optical velocity measurement techniques in fluid mechanics. Exp Fluids. 2002;33:736-42.

[41] http://en.wikipedia.org/wiki/Fog_machine.

[42] Kahler C, Sammler B, Kompenhans J. Generation and control of tracer particles for optical flow investigations in air. Particle Image Velocimetry: Recent Improvements: Springer; 2004. p. 417-26.

[43] Adrian RJ. Laser velocimetry. Fluid mechanics measurements. 1983:155-244.

[44] DYNAMICS D. Dynamic Studio Mannumal. In: communication p., editor.2012.

[45] Theunissen R, Scarano F, Riethmuller ML. Spatially adaptive PIV interrogation based on data ensemble. Experiments in Fluids. 2010;48(5):875-87.

[46] Westerweel J, Scarano F. Universal outlier detection for PIV data. Experiments in Fluids. 2005;39(6):1096-100.

[47] Kitzhofer J, Ergin F, Jaunet V. 2D Least Squares Matching applied to PIV Challenge data (Part 1). 16th Int Symp on Applications of Laser Techniques to Fluid Mechanics Lisbon, Portugal2012.

[48] Brücker C, Hess D, Kitzhofer J. Single-view volumetric PIV via high-resolution scanning, isotropic voxel restructuring and 3D least-squares matching (3D-LSM). Measurement Science and Technology. 2013;24(2):024001. 
[49] Kitzhofer J, Nonn T, Brücker C. Generation and visualization of volumetric PIV data fields. Experiments in Fluids. 2011;51(6):1471-92.

[50] Meslem A, Nastase I, Allard F. Passive mixing control for innovative air diffusion terminal devices for buildings. Building and Environment. 2010;45(12):2679-88.

[51] Meslem A, Dia A, Beghein C, El Hassan M, Nastase I, Vialle P-J. A comparison of three turbulence models for the prediction of parallel lobed jets in perforated panel optimization. Building and Environment. 2011;46(11):2203-19.

[52] Elvsén P-Å, Sandberg M. Buoyant jet in a ventilated room: Velocity field, temperature field and airflow patterns analysed with three different whole-field methods. Building and Environment. 2009;44(1):137-45.

[53] Montoya LD, Jackson JL, Amitay M. Control of aerosol dispersion and removal in a room using synthetic jet actuators. Building and Environment. 2010;45(1):165-75.

[54] Nastase I, Meslem A, Vlad I, Colda I. Lobed grilles for high mixing ventilation-An experimental analysis in a full scale model room. Building and Environment. 2011;46(3):547-55.

[55] Nino E, Fasanella R, Di Tommaso R. Submerged rectangular air jets as a particulate barrier. Building and Environment. 2011;46(11):2375-86.

[56] Sanjuan C, Sánchez MN, Heras MdR, Blanco E. Experimental analysis of natural convection in open joint ventilated façades with 2D PIV. Building and Environment. 2011;46(11):2314-25.

[57] Sanjuan C, Suárez MJ, Blanco E, Heras MdR. Development and experimental validation of a simulation model for open joint ventilated façades. Energy and Buildings. 2011;43(12):3446-56.

[58] Chami N, Zoughaib A. Modeling natural convection in a pitched thermosyphon system in building roofs and experimental validation using particle image velocimetry. Energy and Buildings. 2010;42(8):1267-74.

[59] Kumlutaş D, Karadeniz ZH, Kuru F. Investigation of flow and heat transfer for a split air conditioner indoor unit. Applied Thermal Engineering. 2012;51(1-2):262-72.

[60] Özcan O, Meyer KE, Melikov AK. A visual description of the convective flow field around the head of a human. Journal of Visualization. 2005;8(1):23-31.

[61] Marr D, Glauser M. Length scale propagation along a joint inlet and thermal buoyancy driven flow: Ph. D. thesis; 2007.

[62] Marr D, Khan T, Glauser M, Higuchi H, Zhang J. On particle image velocimetry (piv) measurements in the breathing zone of a thermal breathing manikin. ASHRAE Transactions. 2005;111:299-305.

[63] Marr D, Spitzer I, Glauser M. Anisotropy in the breathing zone of a thermal manikin. Experiments in Fluids. 2008;44(4):661-73.

[64] Eisner A, Rosati J, Wiener R. Experimental and theoretical investigation of particle-laden airflow under a prosthetic mechanical foot in motion. Building and Environment. 2010;45(4):878-86.

[65] Craven BA, Settles GS. A computational and experimental investigation of the human thermal plume. Transactions of the ASME Journal of Fluids Engineering. 2006;128(6):1251-8.

[66] Zhu S, Kato S, Yang J-H. Study on transport characteristics of saliva droplets produced by coughing in a calm indoor environment. Building and Environment. 2006;41(12):1691-702.

[67] Chao C, Wan MP, Morawska L, Johnson GR, Ristovski Z, Hargreaves M, et al. Characterization of expiration air jets and droplet size distributions immediately at the mouth opening. Journal of Aerosol Science. 2009;40(2):122-33. 
[68] Kwon S-B, Park J, Jang J, Cho Y, Park D-S, Kim C, et al. Study on the initial velocity distribution of exhaled air from coughing and speaking. Chemosphere. 2012;87(11):1260-4.

[69] VanSciver M, Miller S, Hertzberg J. Particle image velocimetry of human cough. Aerosol Science and Technology. 2011;45(3):415-22.

[70] Van Hooff T, Blocken B, Defraeye T, Carmeliet J, van Heijst G. PIV measurements and analysis of transitional flow in a reduced-scale model: ventilation by a free plane jet with Coanda effect. Building and Environment. 2012;56:301-13.

[71] Kang J-H, Lee S-J. Improvement of natural ventilation in a large factory building using a louver ventilator. Building and Environment. 2008;43(12):2132-41.

[72] Karava P, Stathopoulos T, Athienitis A. Airflow assessment in cross-ventilated buildings with operable façade elements. Building and Environment. 2011;46(1):266-79.

[73] Rouaud O, Havet M, Solliec C. Influence of external perturbations on a minienvironment: Experimental investigations. Building and Environment. 2004;39(7):863-72.

[74] Posner J, Buchanan C, Dunn-Rankin D. Measurement and prediction of indoor air flow in a model room. Energy and Buildings. 2003;35(5):515-26.

[75] Kobayashi T, Sandberg M, Kotani H, Claesson L. Experimental investigation and CFD analysis of cross-ventilated flow through single room detached house model. Building and Environment. 2010;45(12):2723-34.

[76] Poussou SB, Mazumdar S, Plesniak MW, Sojka PE, Chen Q. Flow and contaminant transport in an airliner cabin induced by a moving body: model experiments and CFD predictions. Atmospheric Environment. 2010;44(24):2830-9.

[77] Liu D, Zhao F-Y, Tang G-F. Non-unique convection in a three-dimensional slot-vented cavity with opposed jets. International Journal of Heat and Mass Transfer. 2010;53(5):1044-56.

[78] Pedersen J, Meyer K. POD analysis of flow structures in a scale model of a ventilated room. Experiments in Fluids. 2002;33(6):940-9.

[79] Sun Y, Zhao Y, Wang X, Zhao L. An algorithm of stereoscopic particle image velocimetry for full-scale room airflow studies. ASHRAE Transactions. 2004;110(1):75-80.

[80] Sun H, Zhao L, Zhang Y. Evaluation of RNG $\kappa-\varepsilon$ and LES non-isothermal models for indoor airflow using PIV measurement data. Transactions of the ASABE. 2007;50(2):621-31.

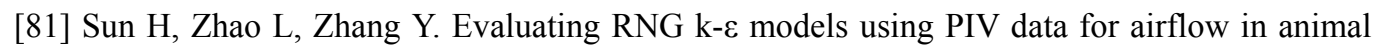
buildings at different ventilation rates. ASHRAE Transactions. 2007;113(1):358-65.

[82] Hosni M, Jones B. Development of a particle image velocimetry for measuring air velocity in large-scale room airflow applications. ASHRAE Transaction. 2002;2:1164-72.

[83] Caciolo M, Stabat P, Marchio D. Full scale experimental study of single-sided ventilation: Analysis of stack and wind effects. Energy and Buildings. 2011;43(7):1765-73.

[84] Mortensen LH, Rode C, Peuhkuri R. Investigation of airflow patterns in a microclimate by particle image velocimetry (PIV). Building and Environment. 2008;43(11):1929-38.

[85] Yang J, Kato S, Nagano H. Measurement of Airflow of Air-Conditioning in a Car with PIV. Journal of Visualization. 2009;12(2):119-30.

[86] Lee JP, Kim HL, Lee SJ. Large-scale PIV measurements of ventilation flow inside the passenger compartment of a real car. Journal of Visualization. 2011;14(4):321-9.

[87] Günther G, Bosbach J, Pennecot J, Wagner C, Lerche T, Gores I. Experimental and numerical simulations of idealized aircraft cabin flows. Aerospace Science and Technology. 2006;10(7):563-73. 
[88] Bosbach J, Pennecot J, Wagner C, Raffel M, Lerche T, Repp S. Experimental and numerical simulations of turbulent ventilation in aircraft cabins. Energy. 2006;31(5):694-705.

[89] Lin C, Wu T, Horstman R, Lebbin P, Hosni M, Jones B, et al. Comparison of large eddy simulation predictions with particle image velocimetry data for the airflow in a generic cabin model. HVAC\&R Research. 2006;12(S3):935-51.

[90] Kühn M, Bosbach J, Wagner C. Experimental parametric study of forced and mixed convection in a passenger aircraft cabin mock-up. Building and Environment. 2009;44(5):961-70.

[91] Wan M, Sze To G, Chao C, Fang L, Melikov A. Modeling the fate of expiratory aerosols and the associated infection risk in an aircraft cabin environment. Aerosol Science and Technology. 2009;43(4):322-43.

[92] Sze To G, Wan M, Chao C, Fang L, Melikov A. Experimental study of dispersion and deposition of expiratory aerosols in aircraft cabins and impact on infectious disease transmission. Aerosol Science and Technology. 2009;43(5):466-85.

[93] Mo H, Hosni M, Jones B. Application of particle image velocimetry for the measurement of the airflow characteristics in an aircraft cabin. ASHRAE Transactions. 2003;109(2):101-10.

[94] Saarenrinne P, Piirto M, Eloranta H. Experiences of turbulence measurement with PIV. Measurement Science and Technology. 2001;12(11):1904.

[95] Westerweel J. Theoretical analysis of the measurement precision in particle image velocimetry. Experiments in Fluids. 2000;29(1):S003-S12.

[96] Gui L, Longo J, Stern F. Biases of PIV measurement of turbulent flow and the masked correlation-based interrogation algorithm. Experiments in Fluids. 2001;30(1):27-35.

[97] Melikov AK, Popiolek Z, Silva M, Care I, Sefker T. Accuracy limitations for low-velocity measurements and draft assessment in rooms. HVAC\&R Research. 2007;13(6):971-86.

[98] Ullum U, Schmidt JJ, Larsen PS, McCluskey D. Statistical analysis and accuracy of PIV data. Journal of Visualization. 1998;1(2):205-16.

[99] Uzol O, Camci C. The effect of sample size, turbulence intensity and the velocity field on the experimental accuracy of ensemble averaged PIV measurements. 4th International Symposium on Particle Image Velocimetry. Germany2001.

[100] Stafford J, Walsh E, Egan V. A statistical analysis for time-averaged turbulent and fluctuating flow fields using Particle Image Velocimetry. Flow Measurement and Instrumentation. 2012;26:1-9.

[101] Theunissen R, Di Sante A, Riethmuller M, Van den Braembussche R. Confidence estimation using dependent circular block bootstrapping: application to the statistical analysis of PIV measurements. Experiments in Fluids. 2008;44(4):591-6. 